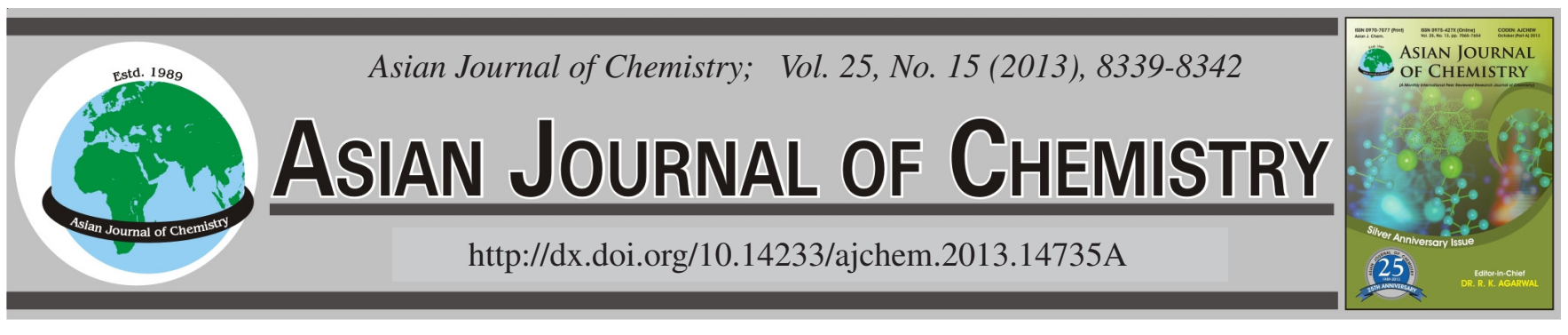

\title{
Optimization Strategy for Isocratic Separation of Halogenated Phenols by Reversed Phase Liquid Chromatography with Methanol-Water Mobile Phase
}

\author{
A. Hakan Aktas* and Aysegül Yilmaz ÇAlik
}

Department of Chemistry, Faculty of Science \& Art, Süleyman Demirel University, 32260 Isparta, Turkey

*Corresponding author: E-mail: ahakan@fef.sdu.edu.tr

\begin{abstract}
Halogenated phenols are priority pollutants. Their determination in drinking waters is, therefore, of great importance and many analytical methods have been developed. Chromatographic methods are suitable for the selective determination of individual phenolic compounds. The aim of this study is to develop a method for separating halogenated phenols such as 3-bromophenol, 3-chlorophenol, 4-fluorophenol, 4-chlorophenol and 4-iodophenol in complex matrices that both sensitive and selective. At the application of HPLC technique in the separation of halogenated phenols, the $\mathrm{pH}$, concentration, in the presence of the water and temperature are important factors affecting its stability. The optimum mobile phase for the chromatographic separation was found to be methanol-water $(50: 50 \mathrm{v} / \mathrm{v})$ with $5 \times 10^{-3} \mathrm{M}$ hexanesulfonic acid, $\mathrm{pH}$ of 4.552 and optimum heat $25^{\circ} \mathrm{C}$.
\end{abstract}

Key Words: Halogenated phenols, HPLC, Separation, $\mathrm{N}_{\mathrm{T}}^{\mathrm{E}}$ solvent strength parameter.

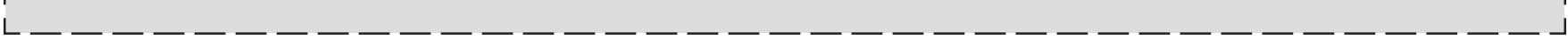

\section{INTRODUCTION}

Methanol is extensively used as a solvent because of its good solvating properties of organic compounds. In addition, its relatively high dielectric solvent $(\varepsilon=32.63)$ allows to solve ionic solutes and prevents ion-pair formation at least at not very high solute concentrations. Among the organic solvents, methanol is the closest to water in structure and properties and, therefore, acidic dissociation in methanol takes place in an analogous solution ${ }^{1}$. Methanol-water mixtures are widely used as solvents in analytical chemistry. Typical uses involve acid-base $\mathrm{pK}$ determination, titrimetric analysis ${ }^{2}$ and HPLC separations $s^{3-5}$. In previous studies, we have demonstrated that the rigorous $\mathrm{pH}$ determination in the mixed solvent used as mobile phases for an HPLC separation of ionizable compounds is needed to get correct retention-mobile phase $\mathrm{pH}$ relationships $^{6}$. In fact, the IUPAC has remarked on the importance of the knowledge of the $\mathrm{pH}$ values of buffers in mixed solvents in order to achieve complete and effective $\mathrm{pH}$ standardization in these media ${ }^{7}$.

Phenols are generated by a number of processes including the petroleum industry, the paper industry and the synthesis of plastics and pharmaceuticals ${ }^{8}$. Halogenated phenols such as 3-chlorophenol or 4-chlorophenol have been used as insecticides and are found in drinking waters as a result of chlorination. Due to their toxicity, the United State Environmental Protection Agency has included some of them in the list of high priority pollutants ${ }^{9}$. Their determination in waste and drinking waters is, therefore, of great importance and many analytical methods have been developed. Chromatographic methods are suitable for the selective determination of individual phenolic compounds, while spectrophotometric methods are employed for the determination of the sum of phenolic compounds ${ }^{10,11}$.

The chromatographer is often concerned with the separation of complex mixtures with a variable behaviour of their components, which makes good resolution sometimes extremely difficult. Several optimization strategies have been proposed to solve this problem ${ }^{12}$. The most reliable and less timeconsuming strategies apply resolution criteria based on empirical or mechanistic models to describe the retention of solutes $^{13}$.

Several HPLC procedures have been reported for the analysis of mixtures of phenols, which are priority pollutants in natural drinking and wastewater ${ }^{14-16}$. Both gas chromatography (GC) and HPLC methods have been reviewed for analyses of phenols in water ${ }^{17}$ and more recently, capillary electrophoresis has also been applied for some separations ${ }^{18,19}$. In general, HPLC methods ${ }^{20,21}$ offer milder conditions for sample preparation than gas chromatography ${ }^{22}$ methods. Therefore, volatile phenols are prevented from being lost. After HPLC separations, target phenols can be identified by means of a diode-array detector ${ }^{23,24}$. Isocratic elution from $\mathrm{C}_{18}$ columns with binary eluents of methanol-water is commonly used ${ }^{15,25}$. 
Quite regularly, however, chromatograms exhibit a lot of additional unidentified peaks, which might include a high number of unknown phenolic compounds.

The Reichardt's NTE scale of solvent polarity of the mobile phase has a primary role in the retention of compounds in reversed phase liquid chromatography ${ }^{26-28}$. The $\mathrm{pH}$ of the mobile phase is another important factor for optimizing selectivity in reversed phase liquid chromatography ${ }^{28}$. In this study the effects of these parameters of on the chromatographic separation were investigated. The true $\mathrm{pH}$ of the mobile phase was taken into account to study the influence of $\mathrm{pH}$ on retention of the compounds ${ }^{29}$. The composition of the mobile phase was optimised by establishing relationships between retention parameters and the scale of solvent polarity at constant $\mathrm{pH}$ of the mobile phase.

The aim of the study was develop a simple isocratic ion pair reversed phase liquid chromatographic procedure for the separation of halogenated phenols.

\section{EXPERIMENTAL}

Solutions of standard samples $\left(0.025 \mathrm{mg} \mathrm{mL}^{-1}\right)$ of 3bromophenol, 3-chlorophenol, 4-fluorophenol, 4-chlorophenol and 4-iodophenol (all Merck, Darmstadt, Germany) were prepared by accurately weighing to $\pm 0.1 \mathrm{mg}$ and dissolving in mobile phase. Stock solution was kept in a deep freezer (at $-18^{\circ} \mathrm{C}$ ). HPLC grade hexanesulfonic acid sodium salt (Fluka, Steinheim, Switzerland) and heptansulfonic acid sodium salt (Fluka) was used as ion-pair reagents. Potassium hydrogenphthalate (Merck, Darmstadt, Germany) was analytical reagent grade.

The HPLC analysis was carried out on a Shimadzu LCVP HPLC system with LC-VP software, a pump (LC-10 ADVP), an auto sampler (SIL-10ADVP) and a diode-array detector (SPD-M 10A VP). A $5 \mu$ M YMC ODS-Pack AM (250 $\mathrm{mm} \times 4.6 \mathrm{~mm}$ I.D.) was used for the analysis.

Measurements of the mobile phase $\mathrm{pH}$ were done with a Mettler Toledo $\mathrm{pH}$ meter using a Hanna combination $\mathrm{pH}$ electrode. Potassium hydrogenphthalate was used as reference material for the standardization of this apparatus in methanolwater binary mixtures in accordance with IUPAC rules.

Chromatographic procedure: The mobile phases containing hexane sulfonic acid sodium salt were prepared by mixing methanol with the aqueous component in the ratio 40:60; 50:50 and 60:40 (v/v). The $\mathrm{pH}$ values of the mobile phases were then adjusted 4.552 with $\mathrm{H}_{3} \mathrm{PO}_{4}$. Chromatographic conditions were as follows; flow rate of $1 \mathrm{~mL} \mathrm{~min}^{-1}$; volume injected $20 \mu \mathrm{L}$; column temperature $25{ }^{\circ} \mathrm{C}$ and detection $280 \mathrm{~nm}$.

The separation column was equilibrated with mobile phase until the baseline was stabilized. Sample injections were made at this point. The time required by the mobile phase to convey a solute from the point of injection onto the stationary phase, through the stationary phase and to the detector is defined as the retention time. The dead time, to, was established for mobile phase tested by injection of a potassium bromide solution in water monitoring the eluate at $210 \mathrm{~nm}$. The capacity factor, $\mathrm{k}$, is a measure of the number of column volumes required to retain a compound. It is defined mathematically as ${ }^{30}$

$$
\mathrm{k}=\frac{\mathrm{t}_{\mathrm{r}}-\mathrm{t}_{\mathrm{o}}}{\mathrm{t}_{\mathrm{o}}}
$$

where $t_{0}=$ is the retention volume of a non retained peak.

The system dead time, $\mathrm{t}_{\mathrm{o}}$, used to calculate capacity factor $\mathrm{k}$, was measured by injecting potassium bromide solution into the system. An average of at least three replicates was used to do all the calculations. Calculation of a value is the division of the $\mathrm{k}$ values of peaks following each other at chromatogram. For instance if the example of 4-fluorophenol and 4-chlorophenol which are two substances succeeding each other is taken into consideration, $\mathrm{k}$ values for these are alternately 2.290 and 5.779. When the $\mathrm{k}$ value of 4-chlorophenol is divided into the $\mathrm{k}$ value of 4-fluorophenol, 5.779/2.290 $=2.523$ is found, which the first value given in Table-1. Also the others are found with the stated calculation.

TABLE-1

RETENTION TIMES, CAPACITY AND SELECTIVITY FACTORS FOR HALOGENATED PHENOLS. MOBILE PHASE:METHANOLWATER (50-50 \% (v/v); pH 4.552; FLOW RATE: $1.0 \mathrm{~mL} \mathrm{~min}^{-1}$; COLUMN: RP $\mathrm{C}_{18}$, LUNA ( $250 \mathrm{~mm} \times 4.6 \mathrm{~mm}, 5 \mu \mathrm{m}$ ID); COLUMN TEMPERATURE: $25^{\circ} \mathrm{C}$; INJECTION VOLUME $20 \mathrm{~mL}$; FOR HALOGENATED PHENOLS $\lambda=280 \mathrm{~nm}$; FOR KBr $\lambda=200 \mathrm{~nm}$

\begin{tabular}{lccc}
\hline \multicolumn{1}{c}{ Elution } & $\mathrm{T}_{\mathrm{r}, \text { ort }}(\mathrm{min})$ & $\mathrm{K}$ & $\mathrm{A}$ \\
\hline 4-Fluorophenol & 8.705 & 2.290 & - \\
4-Chlorophenol & 17.934 & 5.779 & 2.523 \\
3-Chlorophenol & 19.112 & 6.223 & 1.077 \\
3-Bromophenol & 23.145 & 7.747 & 1.245 \\
4-Iodophenol & 30.812 & 10.645 & 1.374 \\
$\mathrm{KBr}$ & $\mathrm{T}_{\text {omean }}: 2.646$ & - & - \\
\hline
\end{tabular}

\section{RESULTS AND DISCUSSION}

$1000 \mathrm{ppm}$ solutions of halogenated phenols were first prepared and absorption scanning between 200 and $900 \mathrm{~nm}$ of wavelengths in UV-visible spectrophotometer and which compound makes maximum absorbance in which wavelength was detected. After we had detected appropriate wavelength, $280 \mathrm{~nm}$ was accepted as common wavelength and we passed HPLC separations in this wavelength.

Separations of compounds which had been studied at different temperatures and the effect of temperature on separation were studied. Standard mixtures of halogenated phenols which had been injected in our studies were prepared and each injection was done according to these concentrations. Concentrations of substances which formed mixture were as follows; 4-fluorophenol 125 ppm, 4-chlorophenol 200 ppm, 3-chlorophenol 100 ppm, 3-bromophenol 50 ppm and 4-iodophenol 525 ppm.

The polarity of the mobile phase is crucial factor that influences solute retention in reversed phase liquid chromatography. Many empirical scales of overall solvent strength have been proposed, but the most widely used is $\mathrm{E}_{\mathrm{T}}(30)$ proposed by Reichardt ${ }^{31}$. Many researches have decisively shown that the plots of $\log \mathrm{k}$ versus the mobile phase $\mathrm{E}_{\mathrm{T}}(30)$ solvatochromic parameter has given better linear correlations than the plots of $\log \mathrm{k}$ versus the mobile phase organic modifier percentage. The normalized $\mathrm{N}_{\mathrm{T}}{ }^{\mathrm{N}}$ parameter is used instead of the $\mathrm{E}_{\mathrm{T}}(30)$ values in order to use similar units to those for the other parameters. Suitable prediction of the retention for a 
specific solute can be achieved from the $\mathrm{N}_{\mathrm{T}}^{\mathrm{E}}$ of the mobile phase and a few experimental data. Therefore, in this work the single solvent polarity parameter, $\mathrm{N}_{\mathrm{T}}{ }^{\mathrm{E}}$ and the relationship with $\log \mathrm{k}$ have been used for determination of optimum separation conditions (Fig. 1). For this purpose, the retention factors for the compounds studied were calculated for three different compositions of the eluent system. Fig. 1 indicates that a good chromatographic separation can be obtained for the halogenated phenols when the methanol content in the mobile phase was $50 \%(\mathrm{v} / \mathrm{v})$. The selectivity and separation factors were appropriate. In chromatographic separation, selectivity factor $(\alpha)$ has an important role in optimization of mobile phase. Variation in selectivity ( $\alpha$ values) versus the organic modifier percentage in the mobile phase is shown in Fig. 2. Hexanesulfonic acid sodium salt was used as an ionpair reagent $\left(5 \times 10^{-3} \mathrm{M}\right)$. This figure shows $\alpha$ for the adjacent solute pairs is suitable for chromatographic separation when the methanol content of the mobile phase is $50 \%(\mathrm{v} / \mathrm{v})$. At the application of HPLC technique in the separation of halogenated phenols, the $\mathrm{pH}$, concentration, in the presence of the water and temperature are crucial factors. The optimum suitable mobile phase at the separation of halogenated phenols that are studied is water that its $\mathrm{pH}$ was adjusted 4.55 values with phosphoric acid and the optimum temperature is $25^{\circ} \mathrm{C}$. Under these circumstances, the capacity factors $(\mathrm{k})$ and selectivity factors $(\alpha)$ were calculated with to help of retention times (Table-1). Chromatogram taking in these conditions is given Fig. 3.

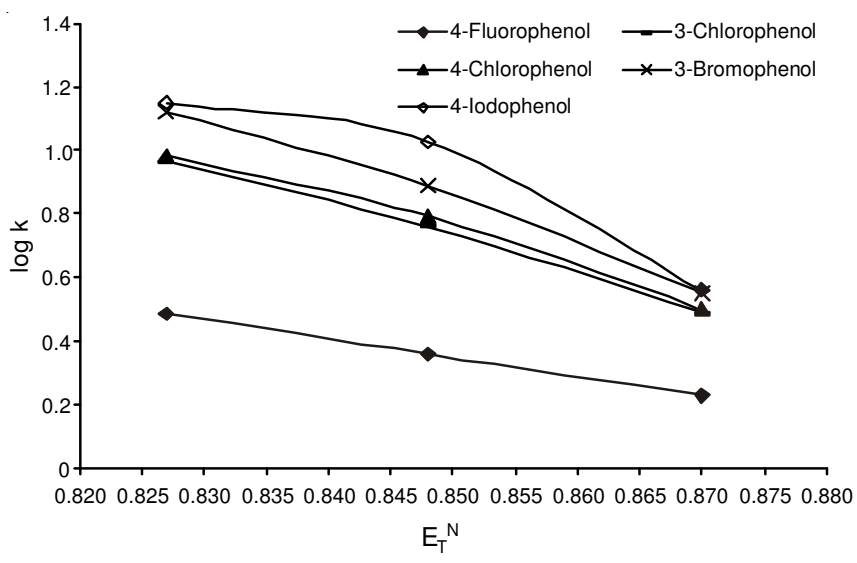

Fig. 1. Plot of $\log \mathrm{k}$ versus single solvent parameter, $\mathrm{E}_{\mathrm{T}}^{\mathrm{E}}$ of mobile phases at 40, 50 and $60 \%$ (v/v) methanol containing hexanesulfonic acid sodium salt at $\mathrm{pH} 4.552$

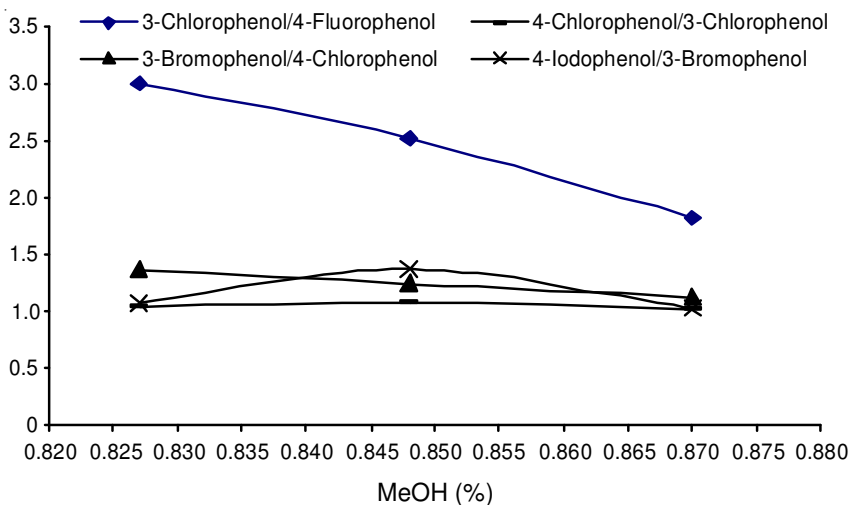

Fig. 2. $\alpha$ values of mobile phases at 40,50 and $60 \%(\mathrm{v} / \mathrm{v})$ methanol containing hexanesulfonic acid sodium salt at $\mathrm{pH} 4.55$

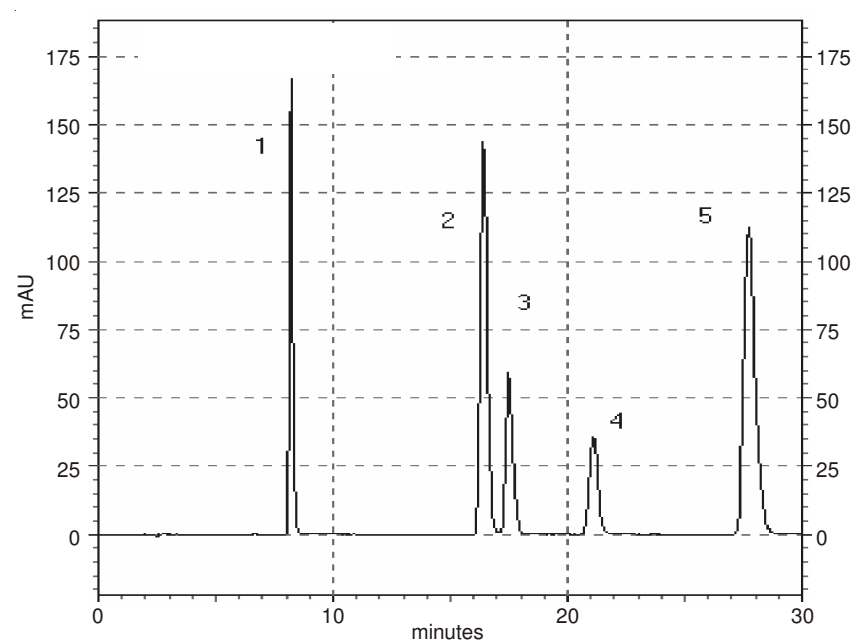

Fig. 3. Chromatogram of halogenated phenols mixture at $25{ }^{\circ} \mathrm{C}$. Peak identification: 1: 4-flurophenol; 2: 4-chlorophenol; 3: 3chlorophenol; 4: 3-bromophenol and 5: 4-iodophenol

After determining the circumstances it was continued to study with $280 \mathrm{~nm}$ wavelength and the next temperatures 30 , 35 and $40{ }^{\circ} \mathrm{C}$ were tried in order to detect the effect of temperature. Increase in the temperature acted as an increase in organic modifier and decrease in retention times was observed. So as to research for the proper temperature conditions, the temperature of $40{ }^{\circ} \mathrm{C}$ were tested which is the highest temperature of the study. After these separation studies it was found that the most proper temperature condition is $25^{\circ} \mathrm{C}$.

After determining the most proper condition and providing sufficient separation, recoveries were calculated for each of the halogenated phenols and analytical data were given in Table-2. As it is seen from the correlation coefficients, with these conditions area-concentration relationship is linear for each compound.

\begin{tabular}{lccc}
\multicolumn{4}{c}{ TABLE-2 } \\
\multicolumn{4}{c}{$\begin{array}{c}\text { RECOVERIES AND ANALYTICAL } \\
\text { DATA FOR HALOGENATED PHENOLS }\end{array}$} \\
\hline $\begin{array}{c}\text { Halogenated } \\
\text { phenol }\end{array}$ & $\mathrm{Y}=\mathrm{a}+\mathrm{bx}$ & $\mathrm{R}$ & $\begin{array}{c}\text { Recovery } \\
(\%)\end{array}$ \\
\hline 4-Fluorophenol & $\mathrm{Y}=12408.8+17477.5 \mathrm{x}$ & 0.999 & 86 \\
4-Chlorophenol & $\mathrm{Y}=1366+12300.5 \mathrm{x}$ & 0.999 & 89 \\
3-Chlorophenol & $\mathrm{Y}=-5007.6+11326 \mathrm{x}$ & 0.999 & 95 \\
3-Bromophenol & $\mathrm{Y}=7495.2+13728.1 \mathrm{x}$ & 0.999 & 88 \\
4-Iodophenol & $\mathrm{Y}=3458.9+5889 . \mathrm{x}$ & 0.999 & 88 \\
\hline
\end{tabular}

Sample working: After optimization of chromatographic separation, drinking water was chosen as the sample for the determination of halogenated phenols (4-fluorophenol, 4-chlorophenol, 3-chlorophenol, 3-bromophenol and 4-iodophenol). $20 \mu \mathrm{L}$ was injected from the sample of mixture, which was distilled with $0.01 \mathrm{M}$ potassium dihydrogen phthalate and at the analysis it was tried to find out the halogenated phenols in the water sample.

At the study, value of 4.552 was obtained for the $\mathrm{pH}$ of halogenated phenols. Upon the injection of this sample to the column, it was seen that halogenated phenols could hold on the column very well and to abolish this condition, $\mathrm{pH}$ was made to pass basic area by distilling the media with $0.01 \mathrm{M}$ potassium dihydrogen phthalate with a distilment rate of $1: 1$. 
For this process Sep-Pac $\mathrm{C}_{18}$ cartridge was used. While 7-8 $\mathrm{mL}$ methanol was being used for the separation studies, in order to minimize the amount of methanol $\mathrm{C}_{18}$ cartridge was tried and it was observed that consumption had fallen to 2-3 $\mathrm{mL}$. By getting out the chromatograms of the parts that was taken with each $1 \mathrm{~mL}$ methanol, detection of halogenated phenols within the water was aimed.

At the study conducted by Hanada et al. $.^{32}, 0.0033-0.0073$ $\mathrm{g}$ was determined as the detection limit for halogenated phenols. By making use of this detection limit, in result of these studies it was determined that no halogenated phenol detection found at the chromatogram of the water sample of the lowest phase.

\section{REFERENCES}

1. F. Rived, M. Roses and E. Bosch, Anal. Chim. Acta, 374, 309 (1998).

2. E.P. Serjeant, Potentiometry and Potentiometric Titrations, Wiley, New York (1984).

3. C.F. Poole and S.K. Poole, Chromatography Today, Elsevier, Amsterdam (1991)

4. E. Bosch, P. Bou, H. Allerman and M. Roses, Anal. Chem., 68, 3651 (1996).

5. M. Roses, H. Canals, H. Allerman, K. Siigur and E. Bosch, Anal. Chem., 68, 4094 (1996).

6. E. Bosch, S. Espinoza and M. Roses, J. Chromatogr. A, 824, 137 (1998).

7. T. Mussini, A.K. Cavington, P. Longhi and S. Rondinini, Pure Appl. Chem., 57, 169 (1985).

8. W. Frenzel, J. Frenzel and J. Moeller, Anal. Chim. Acta, 261, 253 (1992).

9. USEPA, Toxic Substance, Control Act, Washington, DC (1979).

10. F.P. Bigley and R.L. Grob, J. Chromatogr. A, 350, 407 (1985).

11. C.P. Ong, H.K. Lee and S.F.Y. Li, J. Chromatogr. A, 464, 405 (1989).
12. J.R.T. Lapasia, M. Roses, E. Bosch and M.C. Garcia, J. Chromatogr. A, 886, 31 (2000).

13. C.H. Lochmüller, C. Reese, A.J. Aschman and S.J. Breiner, J. Chromatogr. A, 656, 3 (1993).

14. H.K. Lee, S.F.Y. Li and Y.H. Tay, J. Chromatogr. A, 438, 429 (1988).

15. B. Makuch, K. Gazda and M. Karninski, Anal. Chim. Acta, 284, 53 (1993).

16. N. Masque, R.M. Marce and F. Borrull, J. Chromatogr. A, 793, 257 (1983).

17. E. Tesarova and V. Pocakova, Chromatographia, 17, 269 (1983).

18. Y.C. Chao and C.W. Whang, J. Chromatogr. A, 663, 229 (1994).

19. C.D. Gaitonde and P.V. Pathak, J. Chromatogr. A, 514, 389 (1990).

20. C. Baiocchi, M.A. Roggero, D. Giacosa and E. Marenga, J. Chromatogr. Sci., 33, 338 (1995).

21. B. Gawdzik, J. Gawdzik and U. Czwerwinska, J. Chromatogr. A, 509, 135 (1990)

22. I. Cruz and D.E. Wells, J. Anal. Chem., 48, 101 (1992).

23. M.W.F. Nielen, U.A. Brinkman and R.W. Frei, Anal. Chem., 57, 806 (1985).

24. A. Cladera, E. Gomez, J.M. Estela and V. Cerda, Anal. Chim. Acta, 267, 103 (1992).

25. B. Lu, M. Koimur and D. Westerlund, Chromatographia, 46, 72 (1997).

26. B.P. Johnson, M.G. Khaledi and J.G. Dorsey, Anal. Chem., 58, 2354 (1986).

27. J.J. Michels and J.G. Dorsey, J. Chromatogr. A, 457, 85 (1988).

28. P.J. Schoenmakers, S.V. Molle, C.M.G. Hayes and L.G.M. Uunk, Anal. Chim. Acta, 283, 320 (1991).

29. J. Barbosa, R. Berges and V. Sanz Nebot, J. Chromatogr. A, 719, 27 (1996).

30. L. Shelly and S.F. James, J. Chromatogr. A, 964, 91 (2002).

31. C. Reichardt, Chem. Rev., 94, 2319 (1994).

32. Y. Hanada, I. Imaizumi, K. Kido, T. Tanizaki, M. Koga, H. Shiraishi and M. Soma, Anal. Sci., 15, 655 (2002). 